\title{
Le son du TNP de Jean Vilar : les questionnaires des spectateurs (1952-1963)
}

The Jean Vilar's TNP sound : questionnaires of spectators (1952-1963)

\section{Pascale Caemerbeke}

\section{(2) OpenEdition}

\section{Journals}

Édition électronique

URL : http://journals.openedition.org/rsl/1191

DOI : $10.4000 /$ rsl. 1191

ISSN : 2271-6246

Éditeur

Éditions Rue d'Ulm

Référence électronique

Pascale Caemerbeke, «Le son du TNP de Jean Vilar : les questionnaires

des spectateurs (1952-1963) », Revue Sciences/Lettres [En ligne], 5 | 2017, mis en ligne le 02 octobre

2017, consulté le 20 avril 2019. URL : http://journals.openedition.org/rsl/1191 ; DOI : 10.4000/rsl.1191

Ce document a été généré automatiquement le 20 avril 2019.

(c) Revue Sciences/Lettres 


\section{Le son du TNP de Jean Vilar : les questionnaires des spectateurs (1952-1963)}

The Jean Vilar's TNP sound: questionnaires of spectators (1952-1963)

Pascale Caemerbeke

Pourquoi, aujourd'hui, se replonger dans la lecture des questionnaires de spectateurs du TNP de Vilar? N'a-t-on pas déjà tout analysé de ces documents d'archives et tout écrit sur le TNP de Jean Vilar? Que peuvent encore nous livrer ces témoignages, et plus particulièrement, sur le théâtre comme lieu d'écoute ? C'est grâce au projet ECHO que j'ai eu l'opportunité d'explorer ces questionnaires, mentionnés dans les ouvrages sur le TNP et sur Jean Vilar que j'avais lus, et pris conscience que toute cette mémoire écrite avait encore beaucoup à nous dire sur l'évolution du TNP et de ses spectateurs ${ }^{1}$. Dans la présente étude, je me concentrerai cependant sur les remarques concernant le son, afin de réfléchir à l'expérience sonore du spectateur, souvent appréhendée en termes de «trop » ou de " pas assez ». Je présenterai d'abord les questionnaires et leur évolution, puis m'intéresserai aux manifestations sonores du public, enfin à l'équilibre délicat entre voix des acteurs et musique.

\section{À l'écoute des spectateurs: les questionnaires du TNP}

\section{Un échange critique personnalisé}

2 Lorsqu'avant la représentation, le spectateur du TNP parcourt le programme et y découvre le feuillet contenant les questions auxquelles il pourra répondre, celles-ci préparent déjà son écoute, même s'il n'y répond pas. S'il se décide à y répondre, elles l'aident à revenir sur son impression globale et à se remémorer son expérience. Le questionnaire joue ainsi un rôle dans la structuration de la mémoire du spectateur. Il 
permet au spectateur de dissocier des éléments de sa réception (texte, mise en scène, jeu des acteurs, musique) et d'éveiller son esprit critique, de l'affiner au fil des questionnaires, de le légitimer en quelque sorte. Laurent Fleury pointe la fonction « autant symbolique que cognitive ${ }^{2}$ » du questionnaire qui lie chaque spectateur au TNP, et je dirais même à Jean Vilar, puisque le questionnaire lui est adressé personnellement. Le spectateur décline son identité et donne son adresse ; il écrit en son nom à Jean Vilar, c'est une opportunité qui lui est donnée d'établir une correspondance avec un homme de théâtre public. De son côté, Jean Vilar lit effectivement les questionnaires et les annote comme le prouvent les remarques/commentaires qu'il écrit dans les marges; il tâche par la suite de répondre aux critiques ou aux suggestions, autant que faire se peut. Il s'agit donc d'un accord tacite de part et d'autre: le spectateur assume ses propos, que Jean Vilar s'engage à lire. C'est cette interaction qui est l'un des moteurs d'évolution du TNP et des spectateurs en retour, dans un principe dynamique.

En se plongeant dans la vingtaine de boîtes de questionnaires déposée aux Archives nationales ${ }^{3}$, on est à la fois découragé par leur masse et touché par la fraîcheur de ces traces sensibles, livrées spontanément dans l'heure ou les jours qui suivent un spectacle. Chaque questionnaire possède un pouvoir évocateur par la manière dont les propos sont transcrits, par leur style aussi, qui donne des indices sur les spectateurs. Les questionnaires reçus sont tapés à la machine pour composer des livrets ordonnés par rubriques, année après année. Cette pratique chronophage sera abandonnée à partir de la saison 1960-1961, mais les questionnaires seront toujours annotés et classés. Jean Vilar, par l'entremise d'un collaborateur, répondait aussi à certains spectateurs. Dans ce cas, un tampon RÉPONDU est apposé sur le document. Ce peuvent être des remerciements ou des réponses aux questions posées, mais aussi des rectifications lorsque les critiques sont jugées injustes ou disproportionnées. C'est le cas pour cette spectatrice qui reçoit en 1958, à propos de Phèdre, une réponse sèche et ironique de Jean Rouvet, à laquelle elle répondra à son tour, assez dépitée. 
Illustration 1 - Réponse de Jean Rouvet au questionnaire d'une spectatrice de Phèdre en 1958.

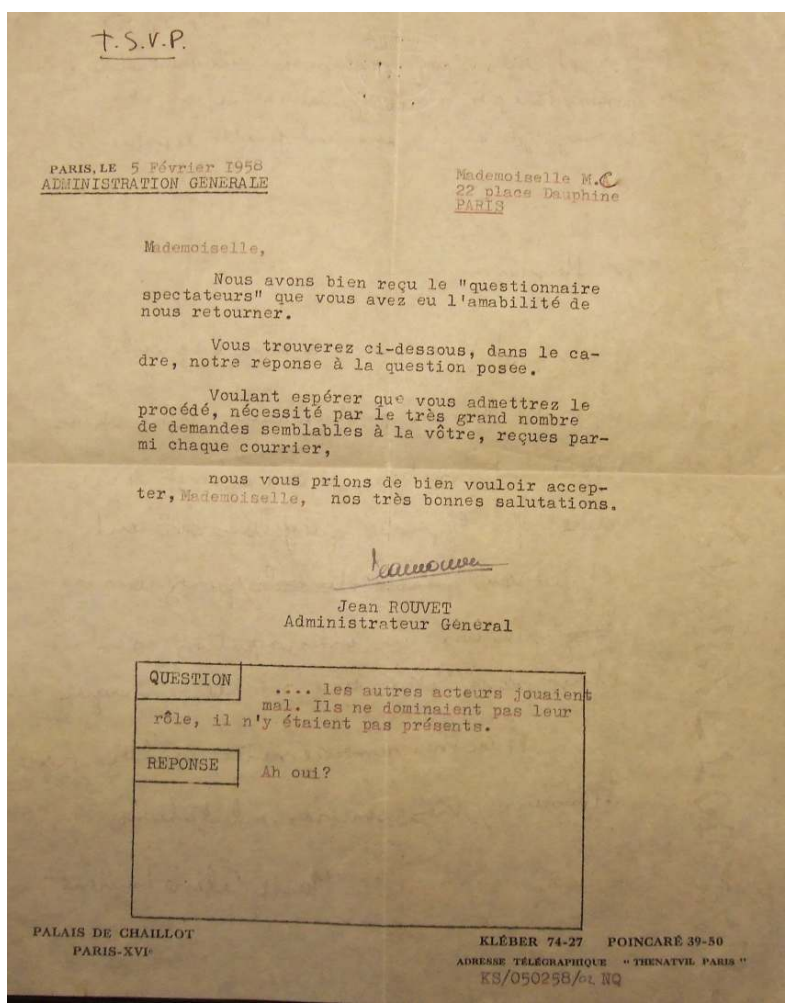

(C)Archives nationales (France). Fonds Théâtre national populaire de Jean Vilar, 295AJ/770-786.

\section{Évolution des questionnaires}

4 Les questionnaires ont évolué au cours des douze années. En 1954, le placement n'est pas précisé, même si certains spectateurs l'indiquent spontanément. Il n'y a pas encore beaucoup de place pour répondre aux quatre questions, qui portent sur le mode de connaissance de la représentation, l'organisation matérielle (location, contrôle, placement, chauffage, etc.), le spectacle lui-même et des suggestions. Dans le questionnaire de l'image ${ }^{\circ} 2$, un spectateur de Ruy Blas, en mars 1954, se plaint des retardataires et conseille de ne les laisser entrer qu'à la fin du premier acte. Jean Vilar inscrit sur le document : « Où en est-on à ce sujet ? ». L'avis sera mis en application dès la saison suivante. De même, face à la suggestion de mettre une boîte pour y glisser le questionnaire au lieu de le poster, Vilar note "Juste » et la mesure sera également mise en œuvre. 
Illustration 2 - Questionnaire d'un spectateur de Ruy Blas, mars 1954.

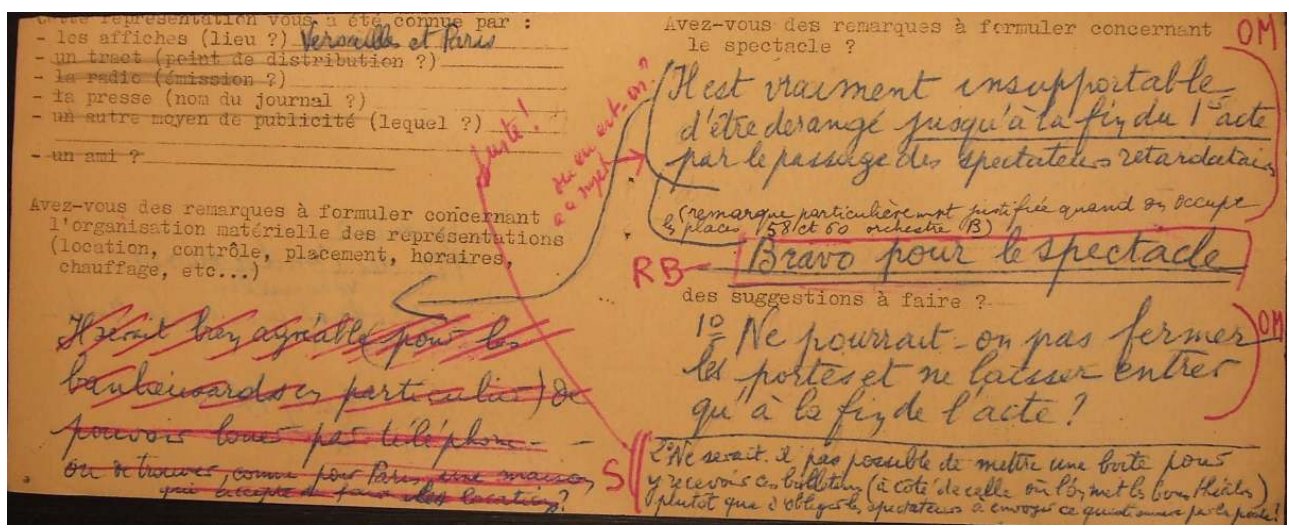

(c)Archives nationales (France). Fonds Théâtre national populaire de Jean Vilar, 295AJ/770-786.

En 1956, les quatre rubriques restent inchangées mais il y a plus de place pour écrire : une spectatrice (cf. image $n^{\circ} 3$ ) en profite pour retracer son parcours et signer sa missive, ignorant les questions. À partir de 1957, le spectateur est invité à inscrire son placement et signe son questionnaire. Il y a désormais huit rubriques au lieu de quatre. Sur les pages extérieures apparaît une question sur la publicité et au dos sont sollicitées les suggestions.

Illustration 3 - Questionnaire d'une spectatrice âgée de 20 ans sur le spectacle Week-end de Noël, 1956.

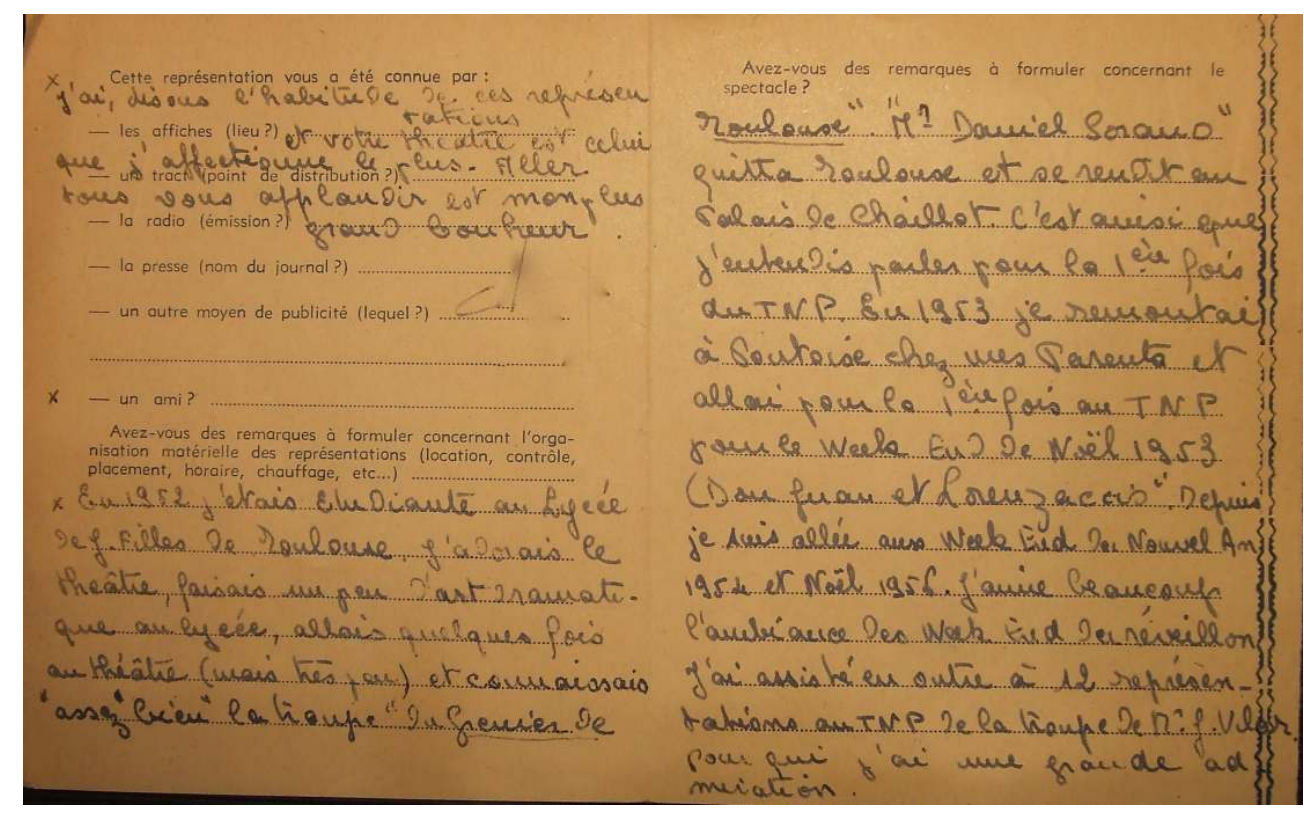

(C)Archives nationales (France). Fonds Théâtre national populaire de Jean Vilar, 295AJ/770-786.

\section{Les « problèmes du "son" »}

6 À l'intérieur du questionnaire figure une question sur la location des places; la rubrique sur l'organisation s'est dédoublée entre organisation de la salle et celle de la représentation; celle concernant le spectacle s'est elle aussi scindée en deux, entre 
l'œuvre représentée, d'une part, sa représentation, d'autre part. Enfin, une rubrique spécifique au son est intégrée, sous l'intitulé "problèmes du "son": l'acoustique, les qualités ou les défauts de l'audition, l'amplification de la musique de scène ».

Il est attesté que la salle de Chaillot était sonorisée dès son inauguration par le TNP le 30 avril 1952, pour la générale publique de L'Avare. Dans une réunion de travail du 24 mars 1952, Maurice Coussonneau est officiellement chargé au TNP de la conduite des appareils stéréophoniques et de tout ce qui concerne le son (sonorisation, amplifications diverses, bruitages, etc.). Des essais de stéréophonie ont d'ailleurs été mis en place par Maurice Jarre, avec l'aide de Bernhardt et Garret, ingénieurs en chef de la Radiodiffusion et inventeurs de la stéréophonie dirigée pour Nucléa ${ }^{4}$, créée le 3 mai 1952. La stéréophonie était utilisée dans d'autres spectacles pour produire un effet de réalité, avec un dispositif supplémentaire pour engendrer une forme d'immersion, comme ce fut le cas dans Le Prince de Hombourg, d'Heinrich von Kleist, créé à Avignon et repris à Chaillot à partir du 7 avril $1952^{5}$.

Dès le début, la sonorisation des spectacles à Chaillot a posé "problème ", comme en témoigne une note de Jean Vilar datée de 1953 : «Je vous rappelle les imperfections de la sonorisation. Il s'agit d'un problème de techniciens et de régies. » Dans une autre note au personnel technique du 6 novembre 1954, Vilar met en garde : «Je tiens à préciser qu'en ce qui concerne la sonorisation des spectacles TNP aucune modification ne peut être apportée aux dispositions arrêtées l'an passé (intensité, place des micros, etc.) sans mon ordre formel. Je compte sur vous pour faire connaitre cette décision nette au personnel L.M.T ${ }^{6}$. Cela est grave. » Jean Rouvet précise enfin dans une note du 22 novembre 1955 : "Les défections actuelles proviennent de la répartition et de la place des amplis qui ont été ajoutés ici et là pour des besoins nouveaux. » En 1956, des travaux d'harmonisation de la sonorisation sont entrepris et annoncés dans la presse. C'est parce que Jean Vilar désirait des retours précis à ce sujet, et dans le contexte d'un énorme chantier technique, qu'il crée la rubrique sur le son en 1957 et demande au spectateur d'indiquer son placement. En décembre 1958, Jean Vilar envoie une note à la régie son : «Voici, relevés sur les questionnaires, les numéros des places occupées par les spectateurs se plaignant de la mauvaise audition du texte... ${ }^{7}$ ».

Un an plus tard, la place dévolue à cette rubrique sera réduite. Aussi une étudiante peutelle écrire en janvier 1958 que « l'acoustique du TNP est parfaite, la meilleure de tous les théâtres de Paris »; tandis qu'une autre fait part de la différence de perception entre ses parents, «qui n'entendent jamais », et elle (cf. image $\mathrm{n}^{\circ} 4$ ). Ces deux étudiantes ont été transportées par la représentation, qu'il s'agisse de Phèdre ou d'Ubu. 


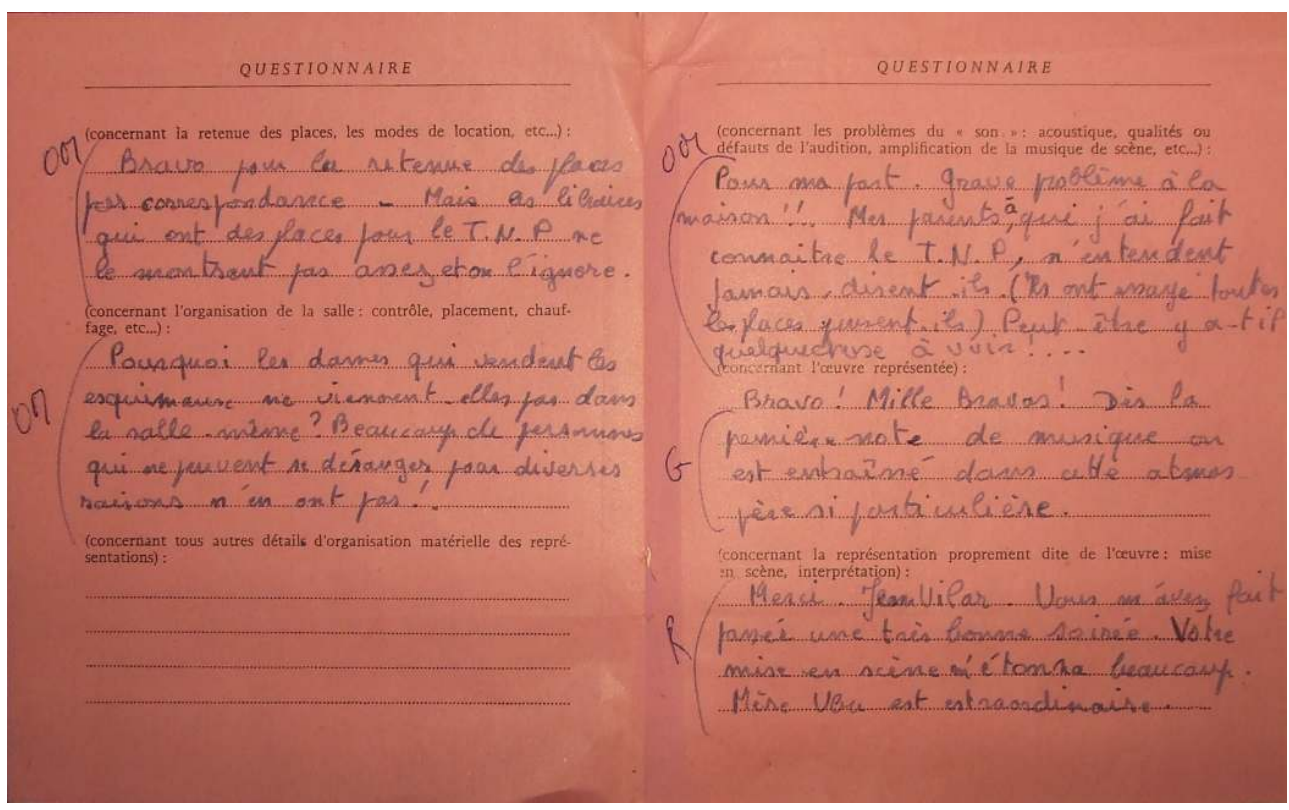

(c)Archives nationales (France). Fonds Théâtre national populaire de Jean Vilar, 295AJ/770-786.

10 Mais, avant même que n'apparaisse cette rubrique sur le son, la mémoire sonore des spectateurs et les commentaires sur l'acoustique sont déjà présents dans les questionnaires. Le palais de Chaillot, qui pouvait accueillir 2700 spectateurs, est souvent jugé comme une salle de spectacle n'étant pas conçue pour le théâtre. Une spectatrice écrit en mars 1954 :

On parle beaucoup ces derniers temps de l'acoustique du palais de Chaillot. Notamment dans le journal Arts où l'auteur d'un certain article la jugeait très mauvaise. J'ai eu des places à $100 \mathrm{Fr}, 200,250$ et 350. Bien au contraire, j'ai eu la surprise agréable de très bien entendre et suivre ce qui se passait sur scène. Ces messieurs de la critique doivent prendre des places à $400 \mathrm{Fr}$ où l'on doit entendre fort mal. Ne peut-on leur proposer des places à $100 \mathrm{Fr}$ ?

Ces exemples nous rappellent le caractère subjectif de la perception du son et de l'acoustique, liée à une forme d'engagement dans l'écoute, à une adhésion au spectacle et/ou au TNP. Vilar, très sensible à la question de l'acoustique, note qu'elle « dépend aussi de la température et de la plus ou moins grande obscuritée ${ }^{8}$ ».

12 Au moment où Vilar annonce qu'il ne renouvellera pas son mandat, en février 1963, un nouveau questionnaire, de type "sociologique», est distribué avec une douzaine de questions liées notamment à la pratique théâtrale. Seule la dernière question demande au spectateur s'il a des remarques à formuler concernant le spectacle auquel il vient d'assister, sans que le son soit mentionné.

\section{Les manifestations du public et les bruits de la salle}

\section{Les applaudissements}

13 Tout de suite apparaissent des commentaires sur les applaudissements, comme celui d'un spectateur venu écouter Lorenzaccio en mars 1952 qui affirme : «Les applaudissements, 
certes mérités, hachent le spectacle, rompent la continuité. Ils détruisent l'atmosphère que la musique peut créer. Ils font, enfin, perdre quelques répliques. Ne peut-on demander aux spectateurs de les réserver pour la fin?" Pour remédier à ces inconvénients, un spectateur de 1953 propose de mettre "des écriteaux: "Interdit d'applaudir avant l'abaissement du rideau"."

14 Jean Vilar s'attaque au problème en 1955. Il écrit aux instituteurs et aux professeurs qui accompagnent des groupes d'élèves à Chaillot pour leur demander de ne plus applaudir pendant le spectacle. Jean-Claude Bardot, dans son livre Jean Vilar, témoigne que "L'avertissement est efficace puisque, lors de la première de Macbeth à Chaillot [le 15 janvier 1955], on siffle ceux qui applaudissent ${ }^{9}$. » La remarque d'une lycéenne en janvier 1959, au sujet des Caprices de Marianne, prouve également que la situation s'améliore de ce point de vue: «Bravo pour votre campagne contre les applaudissements intempestifs, puisqu'on peut maintenant entendre la pièce. Continuez, on pourra peut-être, je le souhaite, entendre la musique !»

\section{Les rires}

Les rires des spectateurs gênent parce qu'ils couvrent des répliques mais aussi parce qu'ils peuvent être le symptôme d'une faute de jeu ou de mise en scène. Une lycéenne venue à L'Avare en 1954 note :

Je trouve que le clerc jouait très bien. Mais qu'il faisait tellement rire qu'on ne pouvait écouter les autres personnages parler. Le clerc devrait faire rire la salle quand les moments ne sont pas très importants. Il devrait faire semblant d'écouter sans trop faire rire quand les moments sont importants.

16 Une autre lycéenne, assistant à Richard II la même année, se plaint que "Gérard Philipe fasse le clown" et se demande "si Shakespeare a réellement voulu faire rire les spectateurs ». Un spectateur d'Arturo Ui en novembre 1960 déclare :

La mise en scène est excellente (comme d'habitude) mais je pense [...] que vous restez trop longtemps dans le grotesque. La salle est forte à $80 \%$ de jeunes pour qui Arturo Ui est un personnage comme Caligula ou Néron. C'est pourquoi la leçon de maintien devrait être grotesque mais ne pas susciter les rires jusqu'à la fin.

\section{Les toux}

Les toux des spectateurs sont évidemment gênantes. Ainsi une spectatrice venue à Dom Juan en février 1954 écrit-elle : «Je reviendrai chaque fois qu'il me sera possible, même si une partie du public est enrhumée et trouble l'audition. » Un spectateur de Phèdre ne rend compte que de cette gêne et suggère de distribuer des pastilles dans la salle et d'en vérifier l'humidification. Toujours en 1958, une étudiante venue assister aux Caprices de Marianne note : «Très bonne acoustique. Très bonne audition des acteurs mais (en est-ce une conséquence?) bruits amplifiés de toux, etc., provenant des spectateurs. » Il semblerait donc que les micros installés pour capter les voix des acteurs, captent aussi les toux de spectateurs près de la scène.

\section{Le bruit des retardataires}

18 La gêne causée par le bruit des retardataires est une critique récurrente. Une spectatrice de Ruy Blas, venue en mars 1954, demande au TNP d'innover en fermant les portes dès le 
début du spectacle. Cela sera fait en 1955 et les retardataires pourront dès 1956 suivre le premier acte sur une télévision installée au bar. Le problème ne semble pas réglé pour autant puisqu'en 1957, une spectatrice du Mariage de Figaro écrit :

Pourquoi fermer les portes au public pour le $1^{\text {er }}$ acte? Sanction contre les retardataires? (en ce cas bravo) [...]. Mais alors pourquoi ne pas faire un véritable entracte après ce premier acte afin de permettre l'entrée de ces retardataires? Car hier soir le $2^{\mathrm{e}}$ acte était commencé et cependant entraient encore des personnes. C'est alors repousser les inconvénients d'un acte. La formule heureuse de fermer les portes ne serait donc qu'une sanction contre les retardataires et ne viserait pas au confort auditif du spectateur installé.

\section{La voix des acteurs et la musique}

\section{Les voix des acteurs, trop faibles ou déformées, altèrent l'intelligibilité du texte} concerne la diction ou le débit qui empêchent de comprendre des pans du texte ; l'autre partie évoque les voix trop faibles, insuffisamment sonorisées ou déformées par la sonorisation. Une spectatrice assistant en novembre 1953 à Lorenzaccio livre d'intéressantes informations concernant les difficultés que rencontre Vilar, et ses incessantes tentatives pour adapter le volume de la salle à la nature des œuvres représentées :

La voix de Jean Vilar, cependant belle, grâce au timbre étendu, me parvenait très mal. Les amplificateurs ne devaient, sans doute, pas être bien réglés, car c'est la première fois que cela se produit pour moi. [...] Le rétrécissement de la salle improvisé pour La Nouvelle Mandragore était une excellente idée. Pourquoi n'avoir pas continué ? Cela enfermait le son dans un espace plus restreint et, malgré le faible organe de G. Philipe, l'on entendait parfaitement, même des loges.

Jean Vilar transmettait souvent les commentaires concernant les voix ou la diction aux acteurs concernés. Ainsi sur le questionnaire d'une spectatrice, venue à Dom Juan en avril 1954, qui écrit à propos de Jean-Pierre Darras: «sa diction exagérément précipitée rendait inintelligible une grande partie de son texte", Jean Vilar note "aviser J.-P. Darras ». Une autre spectatrice témoigne à propos de la Phèdre interprétée par Maria Casarès que l'actrice était inaudible et que les trois quarts du texte ne s'entendaient pas. Cette fois, Jean Vilar ne fait pas de commentaires.

21 De nombreux questionnaires rendent compte des problèmes suscités par les micros et les haut-parleurs sur la transmission des voix des acteurs. Les haut-parleurs produisent parfois « un ronflement perpétuel ${ }^{10}$ ", tandis qu'une spectatrice note que «les micros claquent ». Il leur arrive aussi de faire siffler la voix des acteurs, comme l'atteste cet étudiant de Zurich : "N'ayant rien à reprocher aux spectacles ni aux acteurs, si ce n'est un léger sifflement dans la voix de Gérard Philipe, pourquoi ? Surtout le 12 mars 1954, un des micros se trouvait-il mal réglé ? » Ces plaintes de 1954 résonnent encore en 1958, tout en manifestant l'amélioration technique apportée: «On entend bien mieux qu'avant (pour Mère Courage, c'était moins bon). Cependant quand certains acteurs se déplacent, on s'aperçoit qu'ils quittent un micro : le son n'est pas homogène sur toute la scène ${ }^{11}$. »

Cette observation sur l'absence d'homogénéité du son sur le plateau revient à plusieurs reprises, comme sous la plume de cette spectatrice, la même année 1954: "Dès que 
l'acteur tourne le dos au public, la voix tend à se perdre en une sonorité qui déforme le timbre même de l'acteur le meilleur "; ou de cette autre, commentant une représentation de L'Avare en 1962: «Après ce spectacle, je dois dire merci à Jean Vilar de m'avoir fait découvrir ce que je croyais connaître », et qui note pourtant: "Paroles inaudibles de presque tous les acteurs, lorsqu'ils sont tournés vers la droite au fond de la scène. Dommage. » De telles remarques concernant la nécessité pour les acteurs de jouer face au public pour être audibles de tous, perdurent dans les questionnaires. À l'instar de certains critiques qui feront de ce jeu frontal des acteurs du TNP une caractéristique de l'esthétique et de la dramaturgie villardiennes, on oublie souvent qu'il était dicté par les dimensions et l'acoustique de la salle. Pour terminer sur une note un peu inattendue, notons qu'en 1954, un spectateur suggère qu'il y ait quelques places munies d'un sonotone, comme à la Comédie-Française.

\section{Une émission vocale intempestive : la voix du souffleur}

23 S'il est une voix que l'on ne doit pas entendre, c'est bien celle du souffleur. Un spectateur de Lorenzaccio écrit en mars 1952: "Le souffleur a une excellente élocution. Félicitations (particulièrement goûté l'écho, acte IV, monologue de Lorenzaccio, avant le meurtre).» Tandis qu'une spectatrice de Nucléa de mars 1953 qui a trouvé » remarquable la mise en scène et l'emploi de ces micros dans la salle » suggère " que le souffleur soit plus discret ». Un autre spectateur venu assister à La Mort de Danton en novembre 1953 témoigne :

J'étais placé cette fois à l'orchestre fauteuil 335, tous mes voisins et moi-même nous retournions souvent pour "voir» qui parlait dans notre dos. C'était infernal surtout pendant toute la partie du $1^{\text {er }}$ acte, et à tort ou à raison, j'en ai déduit que c'était le souffleur dont un micro trop sensible envoyait la voix dans la salle.

Jean Vilar note sur le questionnaire : "C'est bien ce que je disais le soir même à Besson. » Enfin, une étudiante s'en prend de nouveau au souffleur à l'occasion d'une représentation de Richard II en 1954 : «il est désagréable que la personne qui souffle souffle sans raison. En effet, plusieurs fois Gérard ne faisait que reprendre sa respiration et on entendait déjà la réplique suivante de la voix du souffleur.» Les critiques concernant cette voix indésirable cessent après 1954, preuve que le problème est résolu, puisqu'il y eut des souffleurs au TNP de 1952 à 1962.

\section{La musique et sa diffusion}

On connaît l'importance de la musique dans les spectacles de Vilar. Elle y était le plus souvent louée, mais tombe souvent sous le reproche d'être ou trop forte ou trop faible. Dans le premier cas, elle couvre « la voix des acteurs par sa grande résonance à travers la salle », comme le note cet étudiant venu voir Nucléa en mars 1953, une remarque que confirme un autre spectateur assistant à une représentation de Lorenzaccio en décembre 1954. Pour Lorenzaccio en effet, une vingtaine de musiciens, dont six trompettistes, jouaient en direct dans la salle. Un spectateur conciliant d'Ubu en avril 1958 conclut par cette formule: «La musique, voyez-vous bien, elle est comme la moutarde de Dijon, excellente et un peu forte. Ceux qui ont les oreilles sensibles en sont un tantinet abasourdis. » À l'inverse, un autre spectateur de La Résistible ascension d'Arturo Ui, en novembre 1962, indique que «La musique de scène, d'une impeccable qualité, a toutefois 
besoin d'être intensifiée. C'est une chose que je répète depuis deux ans déjà, et bien que je sois chaque fois placé différemment, l'audition de la musique de scène me semble toujours trop faible. »

La diffusion joue son rôle dans la gêne parfois éprouvée par le public : « Il est regrettable qu'un spectacle aussi remarquable, à l'interprétation si sûre [...] utilise une sonorisation aussi défectueuse: la musique est diffusée par des haut-parleurs nasillards, grinçant insupportablement dans les aigus ${ }^{12}$ ", d'autant que le TNP compte alors "trente-huit baffles face au public et une quarantaine dans les corniches » selon le témoignage d'un électro-acousticien, spectateur de Mère Courage en 1957. Il déplore la "sonorisation surabondante » qui en découle : "On croit rêver! Et pour diffuser quoi ? La plupart du temps non pas la voix des acteurs [...] mais une "portion de l'orchestre". [...] Il en résulte une cacophonie rocambolesque et déplaisante."

Relevons pour finir une dernière preuve de l'importance de l'expérience sonore au TNP de Vilar. Elle est apportée par les nombreuses remarques, qui s'échelonnent au cours des douze années, concernant les disques édités par le TNP et les demandes précises des spectateurs à ce sujet. Cette spectatrice de Loin de Rueil, au printemps 1961, déplore que la pièce ne soit jouée qu'une quinzaine de jours et demande :

Pourquoi n'y a-t-il plus de disque (33 tours, petit-format) correspondant à chaque pièce jouée cette année ? Pour ma part, j’aimerais retrouver quelques airs (chant, musique de scène) et quelques passages de Loin de Rueil, Roses rouges pour moi, Arturo Ui. Il y a quelques années, le journal Bref informait ses lecteurs à chaque fois qu'un disque de cette sorte était édité...

Si les spectateurs expriment ainsi leurs regrets de ne pouvoir poursuivre l'audition des spectacles en aval, grâce aux disques, ils manifestent aussi leur attachement au rituel préalable à cette audition, celui des trois coups, comme cette spectatrice de Don Juan en avril 1954 affirmant qu'ils " contribuent à une émotion de bon aloi chez le spectateur », et qui déplore leur disparition.

\section{Conclusion}

Il n'est guère besoin de revenir sur la remarquable source d'informations que constituent ces questionnaires de spectateurs du TNP de Vilar. Leur examen a démontré l'importance exemplaire que revêtent les questions d'acoustique et d'écoute au TNP, et l'attention permanente qu'y accordent tant Jean Vilar que son public. Les nombreux aménagements techniques et les tentatives successives de réglage des dispositifs existants en attestent. Sans équivalent connu pour d'autres théâtres ou entreprises dramatiques, ces questionnaires mettent au jour, sous une forme très précise et concrète, la manière directe dont la mémoire sonore des spectateurs du TNP se façonne d'année en année, se formule, et se nuance en fonction des spectacles et des esthétiques mis en œuvre par Vilar et sa troupe. Ils apportent ainsi un complément d'information indispensable aux enquêtes menées dans les archives audio du TNP, ainsi qu'aux études d'architecture et d'acoustique réalisées pour le Palais de Chaillot. 


\section{BIBLIOGRAPHIE}

Bardot, Jean-Claude, Jean Vilar, Paris, Armand Colin, 1991.

Fleury, Laurent, « Le public du TNP et la critique », Sociologie de l'art, Paris, L'Harmattan, 2004, p. 49-77.

Lorcey, Jacques, Notre Jean Vilar, Paris, Éditions Séguier, 2001.

\section{NOTES}

1. De nombreuses questions y sont évoquées, que nous citons sans hiérarchie : heure de début des spectacles; durée des spectacles, avec un ou plusieurs entracte(s); difficulté de faire entrer et sortir plus de 2000 personnes en un temps record; difficultés de location; vie dans le théâtre (tradition du pourboire des ouvreuses qui survit encore quelque temps, serveurs peu aimables, attente au buffet, retardataires, chauffage de la salle); programmes (où le nom des acteurs ne figure pas et que le texte de la pièce et les photos rendent trop chers pour certains); représentations (jeu des acteurs, changements de rôles, costumes, décors, lumières, voix, musique, souffleur); réactions de spectateurs (applaudissements, rires, "éducation des spectateurs ») et leurs relations avec les acteurs après le spectacle; annonces au dos des programmes qui donnent un environnement culturel à la représentation; notes de Vilar, etc.

2. «Deux autres dispositifs (le premier étant les avant-premières) ont contribué à la formation de la fonction critique du public. Le questionnaire d'abord, distribué au début de chaque représentation et destiné à être remis à son issue, participe pour chacun à la reconnaissance de sa qualité de spectateur. L'abonnement ensuite, proposé en début de saison et destiné à fidéliser les spectateurs du TNP sur l'ensemble d'une programmation, fonde la possibilité de juger des spectateurs en les dotant sinon d'une compétence esthétique, du moins d'une identité et d'une mémoire. [...] L'administration systématique de questionnaires auprès de chaque spectateur permet à chaque représentation d'introduire une dimension individuelle dans la relation qui lie le TNP à son public. Le questionnaire ne peut être comparé à la carte d'électeur qui individualise l'expression de la volonté politique; il recouvre pourtant une fonction similaire d'individualisation du jugement, non plus politique, mais esthétique. » (L. Fleury, « Le public du TNP et la critique », p. 63).

3. Les premiers questionnaires datent de février-mars 1952, avant l'installation Jean Vilar au palais de Chaillot le 30 avril 1952, et concernent les avant-premières de Lorenzaccio. Les derniers datent de novembre 1963 et concernent La Vie de Galilée, mise en scène par Georges Wilson, alors que Jean Vilar n'était plus directeur du TNP depuis juillet 1963. Ils couvrent donc toute la période du TNP de Jean Vilar et certains ont été distribués lors de tournées dans des pays francophones, ou durant le festival d'Avignon.

4. En témoigne la lettre de Jean Martinon, des concerts Lamoureux, du 10 mai 1952, adressée à Jean Vilar: "J'ai assisté avec beaucoup d'intérêt à la première de Nucléa et si je souscris médiocrement à la pièce elle-même, je tiens à vous apporter un témoignage de mon admiration pour l'effort de présentation et particulièrement pour la sonorisation, musique et bruitage, absolument efficace principalement dans le premier acte.» (Archives nationales, cote 295AJ/770-786). 
5. « Je mentionnerai enfin que, par l'emploi de la stéréophonie, alors pratiquement à ses débuts, les spectateurs ont été saisis davantage encore, en se retrouvant au cœur de la bataille !» (J. Lorcey, Notre Jean Vilar, p. 32)

6. Le Matériel téléphonique, société absorbée par Thomson-CSF en 1976.

7. Voici la liste des places : 16/11, 513 A, 475 C ; 22/11, 199 B, 234 C, 271 bis A ; 29/11, 301-303 B, 439 A ; 30/11, 20 C ; 4/12, 303 A ; 6/12, 324 A, 492 C ; 7/12, 249 Corbeilles, 37 Corbeilles, 349, 249 Corbeilles.

8. Note en neuf points, de Jean Vilar à Pierre Saveron, le régisseur général, concernant les représentations, du 28 juin au 6 juillet 1952, du Cid et du Prince de Hombourg dans la cour de l'hôtel de Soubise. Voici celles qui concernent le son: «1. Les bruits de la rue [...] 5. Existe-t-il des paravents acoustiques pour mettre sur les murs des côtés, si peu élevés? Voir les murs de la cour d'Avignon. [...] 7. L'acoustique dépend aussi de la température et de la plus ou moins grande obscurité. Exemple : différence très sensible entre l'acoustique de dimanche soir et celle de lundi soir, meilleure lundi soir. "

9. J.-C. Bardot, Jean Vilar, p. 331.

10. Témoignage d'un spectateur, en avril 1961, à propos de Loin de Rueil.

11. Témoignage d'une spectatrice d'Ubu, en 1958.

12. Note d'un spectateur du Mariage de Figaro, 1956-1957.

\section{RÉSUMÉS}

Les questionnaires des spectateurs du TNP de Jean Vilar (1952-1963), qui étaient insérés dans les programmes, ont encore beaucoup à nous apprendre, notamment sur le son. Les remarques des spectateurs sur leur perception auditive sont présentes dès le début, alors même que la rubrique «son » n'apparaît qu'en 1957. Elle sera alors intitulée "Problèmes du "son" : l'acoustique, les qualités ou les défauts de l'audition, l'amplification de la musique de scène ", et témoigne du désir de Jean Vilar d'avoir des retours précis suite aux travaux d'harmonisation de la sonorisation entrepris en 1956. Tout au long de son mandat, Vilar fera tout pour créer les meilleures conditions d'écoute possible, sensible aux remarques des spectateurs sur, par exemple, les manifestations du public, la présence vocale des acteurs et la bonne diffusion de la musique.

The questionnaires for Jean Vilar TNP spectators (1952-1963), which were inserted in the programmes, still have much to teach us, in particular about sound. The spectators' comments on their aural perception were present from the very beginning, even though a «sound » section appeared only in 1957. It was then entitled: "Problems of "sound": acoustics, qualities and flaws of listening, amplification of stage music » and shows Jean Vilar's wish to have precise responses after the works undertaken in 1956 concerning the harmonization of the sound system. Throughout his mandate Vilar will do anything to create the best possible hearing conditions, sensitive as he was to the spectators'comments on, for instance, the public's demonstrations, the actors' vocal presence and the correct diffusion of music. 


\section{INDEX}

Keywords : Jean Vilar, TNP, Chaillot, questionnaires for spectators, acoustics, sonic memory Mots-clés : Jean Vilar, TNP, Palais de Chaillot, questionnaires de spectateurs, acoustique, mémoire sonore

\section{AUTEUR}

\section{PASCALE CAEMERBEKE}

Docteur en anthropologie et études théâtrales, Université Paris 3-Sorbonne Nouvelle et comédienne.

Parmi ses publications :

«La Maison de la radio : un espace de la voix », in G. Faburel et al. (dir.), Soundspaces. Espaces, expériences et politiques du sonore, Rennes, PUR, « Géographie sociale », 2014, p. 89-93.

"Quand la subversion nourrit la norme. La Chambre d'Isabella de Jan Lauwers ", in L. Biscarrat et C. Dussaps (dir.), Normes communiquées, normes communicantes, Revue Essais, Pessac, École doctorale Montaigne-Humanités, 2015, p. 95-107.

«Venir voir Disabled Theater de la troupe HORA. Réflexion sur le handicap mental », in A. Fournier et P. Gilardi (dir.), Annuaire suisse du Théâtre - Mimos « Hora », Berne, Peter Lang Verlag, 2016,

p. 195-205. 